\title{
P-0780-Living with type 2 diabetes in rural Uganda: glycemic control, diet, smoking and alcohol intake
}

\author{
N. Lamichhane', S.K. Bahendeka², D.L. Christensen', J. Nielsen'.
}

\section{University of Copenhagen, Global Health Section- Department of Public Health, Copenhagen, Denmark. 2 MKPGMS Uganda Martyrs University and St. Francis Hospital Nsambya Hospital, Kampala, Uganda.}

\section{Background and Aim:}

Type 2 diabetes (T2D) has become a global health challenge but more so will its effect be on the health of persons living in rural areas of sub-Saharan Africa, where infectious diseases amidst scarce resources present a health challenge. Approaches to the management of T2D include change in lifestyle behaviour such as adapting to healthy and balanced diet, smoking cessation and increased physical activity. Implementation of these treatment measures is challenged by the competing diseases for the scarce resources. Moreover, there is paucity of data on lifestyle behaviour and adherence to diabetes management recommendations in people with T2D in low-income countries. To address this question, we assessed lifestyle behaviour in relation to dietary practices, smoking and alcohol consumption in patients with $T 2 D$ in rural Uganda and their association with glycaemic control.

\section{Method:}

The study population consisted of 45 adults ( 29 males) aged $\geq 40$ years with a laboratory confirmed diagnosis of T2D for at least two years and had attended at least one diabetes education session at a rural hospital in south-western Uganda. Measures of anthropometry, glycated haemoglobin ( $\mathrm{HbAlc}$ ), fasting plasma glucose, and blood pressure were obtained. To assess dietary practices, a household based 7-days Food Frequency Questionnaire (FFQ) was used. To assess adherence to dietary recommendations, a dietary adherence score was developed based on reviews of the International Diabetes Federation's Diabetes Education Training Manual for sub-Saharan Africa, Patient Education documents from Uganda Diabetes Association, American Diabetes Association's nutritional guidelines, and World Health Organization's nutrition guidelines. The adherence score ranged from 0 to 6 and was calculated based on six major dietary components: starchy foods (complex or simple carbohydrate), fruits and vegetables, fat/oil, protein, salt, and alcohol. Glycaemic control was categorized based on individualized target of $\mathrm{HbAlc}$ levels (Ali et al. 2013) including covariates. Linear regression and Chi-square tests were performed to examine various associations.

\section{Results:}

In total, 27 participants (60\%) had HbAlc corresponding to poor diabetes control, and 36 (80\%) had blood pressure levels above $120 / 80 \mathrm{mmHg}$. The major sources of carbohydrates in the diet were cassava and green plantains (matoke). Thirty-nine (88\%) participants consumed cassava 7 days/week and $30(67 \%)$ reported consuming matoke $\geq 6$ days/week. Thirty-eight $(84 \%)$ participants had at least one serving of vegetables and all reported consuming fruits at least two days in a week. However, in all participants, the frequency of consumption of fruits and vegetables was below the recommended'double the frequency of intake of staples'. Palm oil was the most common type of cooking oil (3I participants). For all participants, salt consumption was higher than the WHO recommended $5 \mathrm{~g}$ per person per day. Only one patient smoked and the same patient had a moderate alcohol intake. None of the remaining 44 participants drank alcohol and 12 reported to be former smokers. Using linear regression, we found no association between the dietary adherence score and HbAlc in either the adjusted $(p=0.69)$ (adjusted for age of participants and number of years lived with T2D) or unadjusted model $(p=0.75)$. Further, in chi-square tests, neither glycaemic control nor level of dietary adherence were associated with socioeconomic status or level of education respectively $(p>0.25$ for all tests).

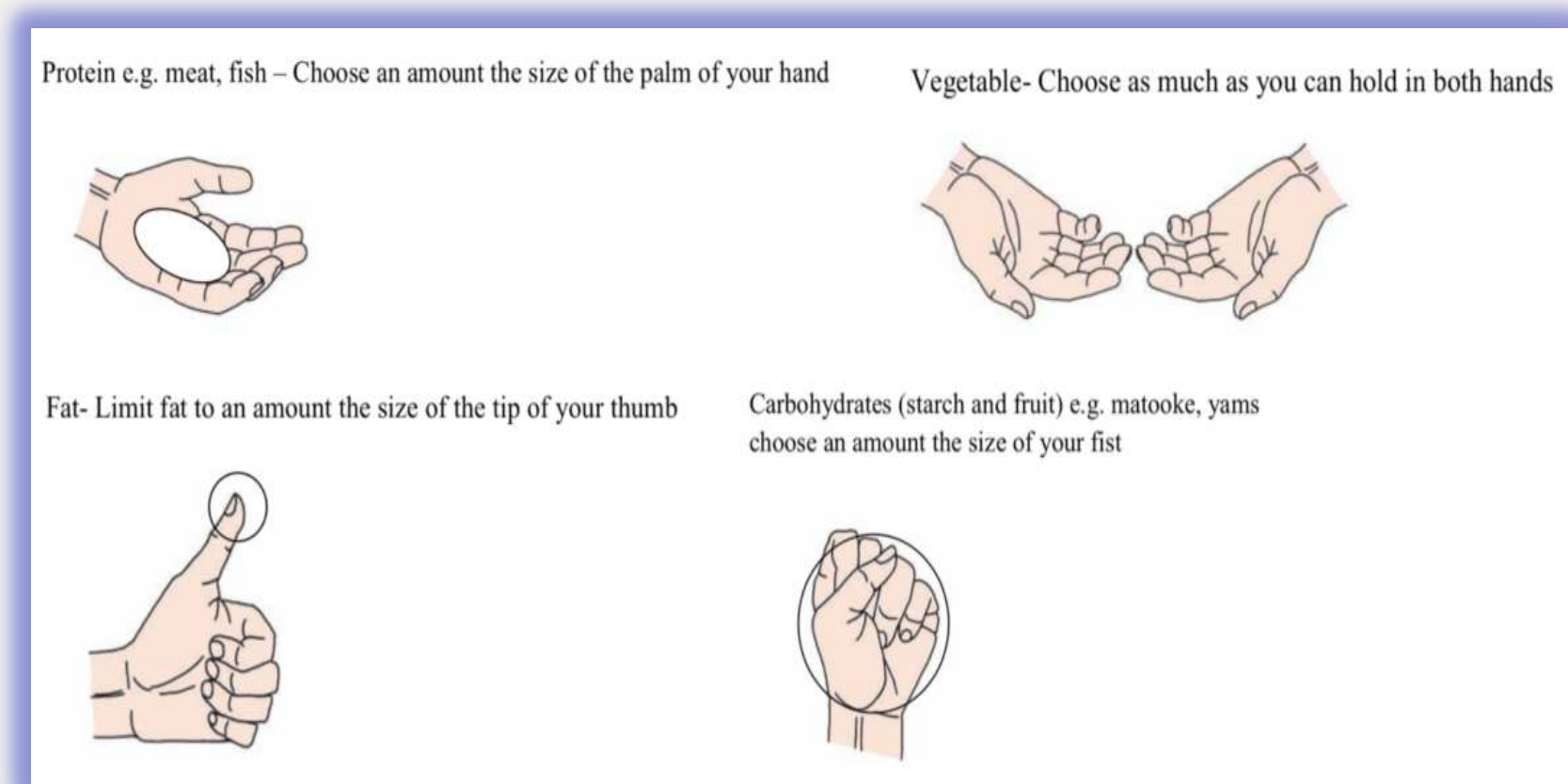

Picture source: $w w w$.ugandadiabetesassociation.org

\section{Discussion:}

The majority of the participants had levels of $\mathrm{HbAlc}$ and blood pressure above the recommendations. Overall, although almost all participants adhered to lifestyle behaviour such as smoking cessation and no alcohol consumption, in contrast, adherence to dietary recommendations was low. Local grown crops like cassava and green plantains dominated the dietary patterns, whereas frequency of intake of fruit and vegetables was very low. As low dietary diversity is commonly reported in Ugandan households, it could be a major barrier to adequate dietary adherence among the study participants. Small sample size, tests of associations not controlled for diabetes medications, and use of household FFQ as a proxy to assess the dietary practices of the participants are major limitations of the study. Future studies with the use of more valid tools to measure quality of diet of people with T2D could help to improve understanding the role of different components of diet in glycaemic control in low-income countries. Therefore, food and nutrition policies should also take into consideration the needs of people living with T2D in rural low-resource settings.

Sources:

- Ali, M. K., Bullard, K. M., Saaddine, J. B., Cowie, C. C., Imperatore, G., \& Gregg, E.W. (20I3). Achievement of Goals in U.S. Diabetes Care, 1999-2010. New England Journal of Medicine, 368(17), 1613-1624. doi:10.1056/NEJMsa I 2 I3829.

- Picture adapted from UGANDA DIABETES ASSOCIATION PATIENTS' PAMPHLET available at: http://www.ugandadiabetesassociation.org/images/UDA\%20patients\%20Pamphlet- 